\title{
10. Hegel y los derechos humanos ${ }^{*}$
}

No resulta fácil hacer una exposición sobre el pensamiento de Hegel con respecto a los derechos humanos, pero la dificultad no estriba en que él no haya elaborado el tema con atención, sino en que su tratamiento es altamente especulativo, es decir, conceptual, y solo de manera indirecta puede ser aplicado a la resolución de los problemas concretos de nuestra vida corriente. Ahora bien, en un programa como este ${ }^{1}$, donde entiendo que se buscan criterios de orientación para confrontar los problemas cada vez más complejos que nos depara el desarrollo científico y tecnológico en su incidencia sobre la vida en general y la vida humana en particular, no me resulta fácil precisar cuáles serían aquellos aspectos de la doctrina hegeliana que pudieran ser más útiles para la reflexión que ustedes llevan a cabo.

En todo caso, voy a tratar de entresacar aquellas ideas que me han parecido de mayor interés para sus expectativas; pero debo adelantarme a decirles que el tratamiento de estas no podrá ser sino inevitablemente abstracto y general, ya

* Esta conferencia fue dictada en un curso de Bioética en la Pontificia Universidad Javeriana, al que fui invitado por su director, el padre Jaime Llano, S. J., en agosto de 2006.

1 Me refiero al curso de bioética dentro del cual se dictó la conferencia. 
que se trata, como lo he señalado, de una reflexión estrictamente filosófica, tal como Hegel entiende la filosofía; es decir, se trata de una reflexión que busca aclarar y precisar los conceptos mismos, para descubrir su fundamentación lógica y su articulación racional.

Voy entonces a comenzar señalando el género de preguntas a las cuales se enfrenta Hegel cuando busca dilucidar el concepto de derechos humanos, recordando que él escribe precisamente en el momento en que se ha llevado a cabo la Revolución francesa, y esta había hecho pública la llamada Declaración de los derechos del hombre y del ciudadano. Por otra parte, no podemos olvidar que, al menos en sus años de juventud, Hegel participó en el entusiasmo con el que una gran parte de los intelectuales de toda Europa recibieron las noticias de la revolución y la instauración de un nuevo régimen republicano. Es cierto que más tarde, a medida en que la revolución tomó un rumbo sangriento y se vio que en cierta forma fracasaba en su intento, Hegel comenzó a distanciarse del fenómeno y trató de comprender las razones de dicho fracaso. Esto es lo que ha llevado a muchos de sus comentaristas a considerar que, con el correr de los años, Hegel abandonó sus ímpetus juveniles y se fue convirtiendo en el filósofo del régimen prusiano.

En una reciente biografía de Hegel, escrita por el conocido filósofo norteamericano Terry Pinkard, leemos el siguiente párrafo con el que comienza su escrito:

Hegel es uno de esos filósofos acerca del cual toda persona educada piensa que sabe algo. Su filosofía fue la antecesora de la teoría de Carlos Marx sobre la historia, pero, a diferencia de Marx quien fue materialista, Hegel fue un idealista en el sentido de que pensaba que la realidad era en último término espiritual, y que se desarrollaba según el proceso de tesis, antítesis y síntesis. También Hegel glorificó al Estado prusiano, diciendo que era obra de Dios, que era perfecto y que venía a ser la culminación de toda la historia humana. Todos los ciudadanos de Prusia le debían obediencia incondicional a ese Estado, el cual 
podía hacer con ellos lo que le viniera en gana. Hegel jugó un papel importante en el crecimiento del nacionalismo alemán, del autoritarismo y del militarismo con sus celebraciones cuasimísticas de lo que él orgullosamente llamo lo Absoluto".

Pero una vez que ha terminado de escribir este párrafo, Pinkard, con muy buen sentido del humor, continúa: "Precisamente todo lo que contiene el párrafo anterior es falso, excepción hecha de la primera frase". Y en párrafo aparte concluye:

Lo que llama todavía más la atención es que todo eso se puede demostrar de manera clara como erróneo, y en los círculos académicos hace ya tiempo que se sabe que lo es, y sin embargo sigue apareciendo en casi toda clase de historias del pensamiento, o en pequeñas enciclopedias que hablan sobre Hegel (Pinkard, 2000, IX).

No voy a detenerme en una reivindicación de la figura de Hegel, que no vendría por supuesto al caso. Pero he traído a colación esta anécdota, porque me parece interesante tenerla en cuenta cuando se pretende comprender las doctrinas sustentadas por él, ya que lo hace casi siempre en un lenguaje no solo muy abstracto y especulativo, sino que da la impresión, al abordar ciertos temas particularmente espinosos, que buscara formulaciones para evitar que sus detractores, que no eran pocos, pudieran utilizarlas en su contra. No podemos olvidar que vivió en tiempos en los que la libertad de expresión se veía amenazada muy fuertemente por parte de los diversos intereses en pugna; intereses tanto políticos como económicos, e incluso religiosos.

Veamos, pues, qué es lo que Hegel nos dice sobre los Derechos humanos, y voy a comenzar por plantear, como lo hace Bernard Bourgeois, dos de las preguntas que van a orientar su reflexión.

1 La primera tiene que ver con el contenido de los derechos, y aquí el asunto es si se trata de una serie compleja y heterogénea de derechos, o si todos ellos 
pueden ser reducidos a un principio básico que los fundamente. Este principio básico tendría que ser una idea del ser humano, una idea sobre lo que significa ser hombre. Pero esto, como puede verse de inmediato, lleva a poner en duda la posibilidad de alcanzar una tal idea que pueda pretender un carácter universal, es decir, que pueda ser válida para todos los seres humanos por encima de sus diferencias culturales, religiosas, filosóficas, étnicas, etc.

Este primer género de preguntas - porque se trata en realidad de eso, no de una simple pregunta sobre qué es el ser humano, sino que ello genera toda una constelación de preguntas complementarias o previas - podría nuclearse alrededor de la pregunta sobre qué es lo que le otorga al ser humano su dignidad, su valor propio. Y aquí las repuestas suelen dividirse entre dos conceptos contrapuestos y en gran medida complementarios. Por una parte se tendría la libertad, y por la otra, la seguridad vital; por un lado tendríamos la dignidad, y por el otro, la felicidad.

Lo que se quiere decir con esto es que no siempre es posible conseguir ambos fines de manera simultánea y resulta, por lo tanto, necesario escoger cuál de los dos tendría prioridad sobre el otro, de modo que el subordinado podría resentirse temporalmente en cuanto a su salvaguarda para asegurar la integridad del subordinante. En otras palabras y de manera muy simple: si para salvaguardar la libertad es posible tolerar un cierto grado de inseguridad y de infelicidad, o si para lograr la seguridad y la felicidad es lícito tolerar un cierto sacrificio de la libertad.

Esto lleva a distinguir entre lo que se ha llamado la libertad formal y la libertad real: en el primer caso, el de la libertad formal, se trata de ofrecer las posibilidades y salvaguardar las condiciones para el ejercicio de la libertad, y en el otro, el de la libertad real, se trata de intervenir de manera activa para que las personas puedan disponer de todo aquello que les asegure su felicidad al menos básica.

Se habla así de unos derechos activos, es decir, aquellos que les permiten a las personas actuar por sí mismas, lograr por su propio esfuerzo sus objetivos, 
y de unos derechos pasivos, que son aquellos en que las personas reciben algo, obtienen ayudas para el disfrute de su derecho a la felicidad. Como podemos ver, se trata en realidad de aquellas ideas que se encuentran en la base de las diferencias que han caracterizado a los partidarios de un liberalismo en general, frente a los promotores de alguna forma de socialismo.

2 Ahora bien, si la determinación de los contenidos que deban tener los llamados "derechos humanos" plantea toda una serie de cuestiones que deben ser analizadas para lograr una respuesta aceptable, otro tanto acontece con la manera en que el ejercicio o la defensa de tales derechos debería llevarse a cabo. Y encontramos aquí una nueva versión del conflicto entre liberales y socialistas - los llamaremos así en aras de simplificar y clarificar los conceptos-, ya que, mientras los primeros defienden la necesidad de salvaguardar a toda costa la libertad como condición indispensable para defender todos los demás derechos, los socialistas consideran que esa posición conduce de manera casi indefectible a la defensa de una libertad formal y vacía, ajena por completo a las condiciones reales de su ejercicio.

Tal vez, en aras de precisar un poco más los conceptos, podríamos visualizar este conflicto sobre cómo salvaguardar los derechos, haciendo intervenir en él a tres actores, a los que llamaremos, respectivamente, liberales, conservadores y socialistas. Cada uno de ellos busca defender como fundamental uno de los tres valores siguientes: los liberales, la libertad; los conservadores, el orden, y los socialistas, la justicia. En una primera aproximación esquemática podemos, entonces, avanzar la siguiente tesis.

Aunque todos convienen en la importancia de esos tres valores para la vida social, los liberales consideran que si no se salvaguarda la libertad como condición de todos los demás derechos, estos mismos derechos, y por supuesto la misma libertad, terminan por sucumbir. Es el ideal que movió durante largos años las luchas de los librepensadores por la instauración de un régimen de libertades que garantizara la obtención paulatina de la igualdad y el orden. Solo a través del ejercicio de la libertad, que constituye la naturaleza más profunda del ser humano, resulta posible lograr el disfrute de los demás derechos 
sin que ello venga a redundar en un detrimento a la dignidad humana. Perdida la libertad, el orden se convierte en represión y la felicidad en abyección.

Los conservadores, por su parte, consideran que si no se mantiene el orden social básico, se termina cayendo en el caos, en el que viene a reinar el más fuerte, con lo cual se pierde la libertad y se sacrifica la justicia. Es, en el fondo, la idea que encontramos en la doctrina política de Hobbes: los seres humanos, dejados en su estado natural, buscan defender sus intereses personales, y como disponen de una inteligencia, logran mirar más allá del presente. Esa mirada les permite considerar a todos sus semejantes como posibles contrincantes, y esto lleva a convertirlos a todos en potenciales enemigos. Es entonces la guerra de todos contra todos, de la cual solo es posible escapar mediante la constitución de un gobierno fuerte, capaz de poner orden y mantener la paz.

Los socialistas, por su parte, consideran que la justicia debe preceder a los demás derechos, porque de lo contrario esos derechos terminan siendo puramente formales, es decir, vacíos y carentes de toda efectividad. El ejemplo aquí podría ser Karl Marx, quien coloca en la base de las relaciones sociales la fuerza de la economía. Es necesario establecer una justicia general, así se tenga que sacrificar temporalmente el orden y la libertad. Por eso, algunos llegan hasta defender el derecho a la revolución, es decir, el empleo de la violencia.

Ahora bien, es importante tener en cuenta que, en principio, las tres formas de pensamiento político comparten la idea de que esos tres valores son fundamentales para la vida en sociedad. La diferencia se halla en la forma de priorizarlos a la hora de ponerlos en marcha, ya que no parece posible lograrlos todos a la vez y de forma completa.

Cabe notar que, una vez que la esperanza de una revolución desaparece del horizonte, ya sea por evaluación de las condiciones reales que no la muestran como posible, ya sea por principios que niegan el derecho a realizarla, las divisiones dentro del juego democrático tienden a reducirse en principio a dos: los liberales y los socialistas. Esto comporta naturales inconvenientes, porque 
desemboca en una división bipartita, clásica en el pensamiento maniqueo, ya que permite diferenciar de forma simple y en apariencia muy clara entre quienes tienen razón y quienes no la tienen, entre buenos y malos. Por supuesto que los que tienen razón y son buenos serán siempre aquellos que comparten el propio punto de vista de quien utiliza esa peligrosa dicotomía.

A su vez, si tenemos en cuenta que históricamente los adversarios del liberalismo han sido los conservadores, veríamos a los socialistas convertidos en los nuevos conservadores, lo que, por supuesto, no parece una forma correcta de interpretar el espectro político

Todo esto ilustra muy bien cómo la determinación de los derechos humanos comporta de manera inevitable problemas de índole política que no pueden ser soslayados. En realidad, la discusión sobre el sentido de los derechos humanos y su efectiva defensa es un tema radicalmente político. Por otra parte, como lo señala muy bien Hegel, y tendré ocasión de examinarlo más adelante, los derechos humanos son el fundamento de los derechos ciudadanos, pero en la práctica son los derechos ciudadanos los que hacen que los derechos humanos lleguen a ser efectivos.

Ahora bien, si nos fijamos en un nivel más conceptual, cabe señalar una significativa diferencia entre el derecho y la felicidad, porque el derecho implica, por su misma naturaleza, una distinción básica entre lo que es y lo que debe ser, es decir, el derecho establece una distinción entre él mismo y lo real, mientras que la felicidad busca integrar ambos momentos, más aún, identificarlos. Vista así la diferencia entre estos dos conceptos, podría decirse que un socialista pretende integrar ambos momentos, y hacerlo en beneficio de la identidad, es decir, de la felicidad, lo que conduciría a un socialismo democrático que privilegia la obtención de la felicidad aun a riesgo de tener que limitar las libertades. Los liberales, en cambio, consideran que se debe privilegiar la diferencia, es decir, el derecho, dándole así primacía a los derechos activos sobre los pasivos, pero sin descuidar estos, claro está. Como he tenido ocasión de precisar, se trata de una ordenación de los derechos, sabiendo que deberán buscarse todos, pero que no resulta posible hacerlo de manera 
simultánea, y esto exige establecer un orden de prioridades para resolver los casos conflictivos.

Ahora bien, si después de estas consideraciones de carácter general y de principio, regresamos al evento inaugural de los Derechos del hombre y del ciudadano, como fue su proclamación en 1789 como preámbulo a la Constitución de 1791, podemos considerar, como lo hace Bernard Bourgeois, que los tres grandes pensadores que buscaron analizar esos derechos desde la filosofía fueron Immanuel Kant, Johann Gottlieb Fichte y Georg Wilhelm Friedrich Hegel. En una primera visión de conjunto, podemos sintetizar sus posiciones diciendo que todos tuvieron los ojos puestos sobre la libertad como el carácter fundamental del ser humano, y como aquello que caracteriza el advenimiento de la nueva época, la llamada Modernidad. En otras palabras, los tres fueron, cada uno a su manera, liberales.

Cabe recordar que, en la visión histórica de Hegel, la Modernidad, en el orden del pensamiento, había tenido su comienzo con la filosofía de René Descartes, en el siglo XVII, y había llegado a su plena manifestación con la filosofía crítica de Kant. Mientras que, en el orden de la realidad política, había comenzado con la Reforma protestante en el siglo XVI y había llegado a su culminación con la Revolución francesa. Se trataba, entonces, de comprender ese doble fenómeno conceptual e histórico para tratar de encontrarle sus verdaderas raíces y su significado.

Ahora bien, como culminación del pensamiento moderno, Kant había desarrollado una filosofía que se proponía subordinar la función teórica de la razón humana a su función práctica, es decir, poner el conocimiento al servicio de la moral. Con ello buscaba mostrar cómo, por una parte, las condiciones que debe cumplir el ejercicio teórico de la razón mediante el entendimiento, es decir, las condiciones para hacer ciencia, descalifican al entendimiento para juzgar sobre su ejercicio práctico. En otras palabras, que las ciencias no tienen competencia para convertirse en juez de la moral. Por otra parte, Kant señala igualmente que el ejercicio de la función práctica de la razón, es decir, la tarea de orientar el propio comportamiento a la luz de la razón, 
debe llevarse a cabo a partir de la pura reflexión de la razón sobre sí misma y sus condiciones de posibilidad prácticas. Esto, porque se trata de establecer principios de comportamiento que sean válidos para todos los seres humanos en todas las circunstancias, es decir, de elaborar una moral realmente universal.

Ahora bien, el resultado último de esta propuesta kantiana, si bien conduce a una radical autonomía en el ejercicio práctico de la razón al desligarlo de su ejercicio teórico puramente cognoscitivo, trae a su vez consigo una doctrina moral de carácter exclusivamente formal, vacía, alejada de la realidad, y por ello mismo incapaz de responder en forma adecuada a los retos concretos que plantea el obrar humano en su realidad cotidiana. Esto se debe a que, al colocar el ejercicio práctico de la razón por encima de su ejercicio teórico, la naturaleza, que viene a ser el objeto de este ejercicio teórico, se ve supeditada a la libertad como carácter propio de la razón práctica. Expresado en términos concretos, esto significa que el ejercicio de la libertad no se lleva a cabo dentro de los parámetros del mundo empírico, del mundo tal como nos es dado, sino que tiene lugar en el campo de la pura conciencia, en el interior de esta. La moral viene a convertirse así en un fenómeno subjetivo, interior a la conciencia, lo que lleva a una moral rigorista que no tiene en cuenta las condiciones reales de su ejercicio, y que muestra un rechazo a priori a la injerencia de las inclinaciones naturales de los seres humanos.

Nadie puede negar que la moral kantiana es muy elevada, muy exigente y hasta muy racional, pero sucede igualmente que es meramente formal, vacía de contenidos, abstracta e irrealizable. Por eso, para tratar de corregir ese formalismo vacío, Fichte propone que, en vez de separar el ejercicio teórico de la razón de su ejercicio práctico, como lo hace Kant, se invierta más bien la relación que suele establecer el sentido común. En efecto, para el sentido común, le corresponde al ejercicio teórico establecer normas definitivas a las que deberá someterse la razón en su ejercicio práctico. La propuesta de Fichte es que se derive lo teórico de lo práctico, para lo cual hay que mostrar cómo, ya en sus mismas raíces, el ser humano se constituye como pensante precisamente porque es un ser con voluntad, un ser práctico. 
Si recordamos a Descartes, él nos había mostrado cómo la primera verdad realmente indubitable es la que tiene el pensamiento cuando piensa en su propia existencia: cogito, sum. El pensamiento no puede dudar de su existencia sin que por ello mismo la afirme. Pero si bien esto es muy cierto, Fichte hace notar que cogito (pienso) es un acto de pensar, y por ello mismo es ante todo un acto, es decir, una acción de la voluntad. Antes que un mero yo pienso, se trata en realidad de un yo quiero pensar. Es ese acto el que se halla en el origen de la conciencia y de su diferenciación frente el mundo, de modo que el carácter de ser pensante, que la sitúa a la conciencia frente al mundo al saber de sí misma y del mundo como de algo distinto de ella, proviene en realidad de su condición de ser capaz de querer, es decir, de su voluntad.

Partiendo de esa importante constatación, a saber, de que el pensar es originariamente un acto de la voluntad, busca Fichte justificar y fundamentar todos los esfuerzos humanos para conocer la realidad, al entenderlos como otros tantos esfuerzos para responder a la pregunta fundamental que había señalado Kant: ¿qué debo hacer? De esta manera, las ciencias vienen a convertirse en "siervas de la moral", en sus servidoras, al ofrecerle las condiciones para su ejercicio.

Sin embargo, Hegel considera que con esa inversión que viene a fundamentar el ejercicio teórico de la razón sobre su ejercicio práctico, Fichte no logra superar el formalismo kantiano, porque continúa otorgándole prioridad a la reflexión especulativa sobre la realidad concreta. Su visión no pasa de ser una especie de utopía inalcanzable que termina por despreciar el mundo de la realidad concreta, al pretender reducir la realidad a un simple medio para la realización de la libertad, y al buscar imponerle pautas a las que esa realidad no puede responder. La realidad parece condenada a no ser otra cosa que una inevitable traición a los principios ideales de la moralidad, un eterno deber ser inalcanzable.

Hegel se propone, entonces, alcanzar una verdadera síntesis entre el ejercicio teórico y el ejercicio práctico de la razón, entre el conocimiento y la libertad, entre el ser y el deber ser. Para ello, busca sobrepasar lo que considera 
el punto de partida erróneo de esas dos filosofías, la de Kant y la de Fichte, punto de partida al que se refiere como "principio de la reflexión", y que ya Reinhold había intentado establecer como fundamento de toda filosofía crítica. Este principio señala como condición insuperable de todo saber la distinción originaria entre el sujeto y el objeto, entre el pensamiento y el ser, de modo que no es el pensamiento mismo el que establece la diferencia entre el pensante y lo pensado, sino que estos dos vendrían a preceder el acto mismo de pensar.

Esta forma de pensar, propia del sentido común, considera que sujeto y objeto existen previamente al acto de conocer y lo condicionan, lo que conduce a ver lo ideal, es decir, lo propio del pensamiento, como una meta inalcanzable que la realidad debería tomar como modelo. Esto implica una desvalorización de lo real, ya que no pasa de ser un pobre remedo de su ideal, como sucedía ya en el caso de Platón. Si la verdad es lo ideal como tal, lo real no puede ser más que su imagen deformada y despreciable. Por eso Hegel, al insistir en que es el acto mismo de pensar, es decir, de tomar conciencia, el que establece la diferenciación entre el sujeto y el objeto, entiende que la realidad no es simplemente lo dado, lo que está ahí antes de todo proceso cognoscitivo, sino el resultado de la interacción cognoscitiva que permite que esa realidad manifieste su articulación racional. De ahí que lo real no sea, para Hegel, lo simplemente dado, sino precisamente aquello que se manifiesta como resultado de la reflexión humana sobre lo dado; porque lo dado como tal es algo pasajero y inconsistente que solo gracias a la conciencia logra manifestar su articulación racional. Por eso, lo que solemos llamar la realidad viene a ser propiamente el fenómeno de esa articulación racional de su devenir.

Ahora bien, esas tres filosofias, las de Kant, Fichte y Schelling, tienen, sin embargo, algo muy importante en común, y es que ellas establecen la libertad como principio de confluencia conceptual o lógica de todos los derechos que tiene o pueda llegar a tener el ser humano. Son, como hemos dicho, radicalmente liberales, de modo que, en lugar de mezclar de manera empírica los diversos derechos humanos, vienen a fundamentarlos en la libertad misma como aquel derecho originario que busca en los demás derechos su 
manifestación exterior. En esta forma, mientras que la Declaración de los derechos del hombre y del ciudadano de 1789 yuxtaponía sin un orden claro los diversos derechos, como libertad, propiedad, seguridad, resistencia a la opresión, etc., estos filósofos lo que buscan es encontrar la fuente o fundamento de todos ellos en la libertad del ser humano en virtud de su carácter de ser consciente, es decir, de aquel que sabe de sí y sabe de lo otro de sí, más aún, que sabe de lo otro de sí porque sabe de sí mismo.

Es así como la libertad no viene a ser en realidad un derecho más para el hombre, algo que se le otorga por parte de los demás, de la sociedad o del Estado, sino el principio actuante mediante el cual el hombre mismo se otorga a sí mismos su derechos. Como lo ha expresado Bernard Bourgeois, "el hombre no posee plenamente los derechos, si él mismo no se los otorga: el derecho solo existe como autoafirmación de la libertad, solo existe en la libre declaración de sí mismo" (17-18).

La tarea consiste, entonces, en elevar a concepto, es decir, en descubrir el sentido lógico o racional, y por tanto necesario y universal, de esa declaración de derechos; porque esa comprensión es la que viene a otorgarle fuerza a la reivindicación de tales derechos, sin la cual su otorgamiento no pasa de ser una ficción vacía. La función del Estado no puede ser, por tanto, la de velar por la felicidad de los súbditos, sino la de garantizarles el pleno ejercicio de su libertad. Lo expresa muy claramente Fichte cuando escribe: “¡No, Príncipe, tú no eres nuestro Dios! De Él esperamos nuestra felicidad, de $t i$, en cambio, solo esperamos la protección de nuestros de derechos. ¡Tú no debes ser bueno con nosotros, sino justo!" (citado por Bourgeois, 18).

Si nos detenemos ahora en Hegel y en su propuesta para fundamentar la reivindicación de los derechos humanos, cabe recordar que su entusiasmo por el hecho revolucionario francés fue adquiriendo con el tiempo rasgos cada vez más críticos. En verdad, su concepción de la realidad en general fue volviéndose cada vez más histórica, de modo que viene a considerar el ejercicio revolucionario y su fracaso como una forma inadecuada de aplicar la teoría a la práctica, como una realización no mediada de la idea de libertad. 
Esto, por supuesto, no implica descalificar las pretensiones revolucionarias, sino comprender la necesidad de un proceso mediador que permita realizarlas sin destruir por ello la realidad.

Comienza, entonces, por rechazar la idea de convertir los derechos del hombre en el fundamento mismo del derecho, o en su principio absoluto. El verdadero orden conceptual establece la libertad como ese principio, por constituir ella la naturaleza propia del ser humano. La persona se hace real mediante su propia autoposición, y se realiza en búsqueda de su utilidad en el seno de la sociedad en la que vive. Esa libertad tiene su realización suprema en la obediencia del ciudadano a las leyes en cuya elaboración él mismo ha tomado parte. En otras palabras, y como lo señalamos antes, los derechos humanos se fundamentan en la libertad, pero esta solo llega a ser real dentro del Estado, es decir, solo se vuelve realidad gracias a los derechos civiles o del ciudadano.

El primer momento, el de la libertad, caracteriza al ser humano como voluntad pura, es decir, no como voluntad que quiere esto o aquello, sino como voluntad que se quiere a sí misma, como voluntad que es capaz de sobrepasar toda determinación o condición concreta. Ahora bien, esta libertad se mantiene abstracta o simplemente posible e inefectiva, mientras no llegue a su ejercicio real en el seno de una sociedad dentro de la cual los individuos buscan ser reconocidos como libres. Pero ese ejercicio de la libertad, que le permite al individuo buscar sus propios intereses y establece así un proceso de diversificación (estamentos, profesiones, diferencias económicas, etc.), solo puede hacerse efectivo en el marco de una legislación que salvaguarde dicho ejercicio. Por eso, si los derechos humanos vienen a hacerse reales gracias a los derechos del ciudadano, estos a su vez tienen su fundamento en aquellos: moral y política juegan así una doble relación. La moral fundamenta la política, mientras que la política hace efectiva la moral. Y esto explica por qué Hegel considera que la instauración del Estado, es decir, del Estado de derecho - que no debe confundirse con el gobierno-, viene a convertirse en la meta final del ejercicio de la libertad. 
Es importante tener en cuenta que los derechos humanos no necesitan ser declarados para tener vigencia, sino que existen como tales con la sola existencia de seres humanos dotados de libertad. Ahora bien, como su realización está condicionada por los derechos civiles o del ciudadano, y estos no existen por naturaleza sino en virtud de su configuración social, es necesario prestar atención a la constitución de estos últimos. De esa manera, en el nuevo orden político republicano, que estaba tomando cuerpo en Europa, Hegel veía la realización concreta de lo que en su origen había sido un principio cristiano, al menos tal como lo concibió la Reforma protestante: en su exclusivo sometimiento del ser humano a la voluntad divina tal como esta se revela mediante la Sagrada Escritura, el ser humano alcanza su absoluta libertad frente a todos los poderes finitos. Como lo expresaba Martín Lutero en su escrito sobre La libertad cristiana, es la total servidumbre del hombre a Dios la que lo convierte en un ser absolutamente libre. No olvidemos que la servidumbre con respecto a Dios viene a ser la total obediencia a la propia conciencia.

De ahí que Hegel, como luterano convencido, establezca un fuerte contraste entre la vivencia del mensaje cristiano tal como la ha llevado a cabo el catolicismo romano durante la Edad Media, y su nueva comprensión gracias a la Reforma iniciada por Lutero, contraste que explica en buena medida el paso del orden medieval al nuevo orden político que instaura la modernidad. Sus convicciones luteranas llevaron a Hegel a elaborar una dura crítica contra el catolicismo romano, pero su visión histórica lo hace ver el fenómeno del catolicismo, no como una simple deformación del mensaje cristiano, sino como un periodo por el que fue necesario pasar para convertir ese mensaje en realidad espiritual, es decir, hacerlo comprensible a la razón. Para Hegel, como filósofo de la historia, ese periodo juega un papel determinante en el desarrollo del espíritu, es decir, de aquella realidad que da sentido a lo que existe. El catolicismo corresponde al periodo de educación de los pueblos europeos, educación que debió someterlos a una dura disciplina necesaria para que aprendieran a comprender de manera adecuada lo que Dios les había revelado. Es cierto que, para Hegel, esa forma de cristianismo en el mundo moderno viene a mostrarse anacrónica, propia de una época ya pasada en la que jugó un papel necesario pero por ello mismo superado. 
Hegel lee la historia del cristianismo en tres grandes etapas. La primera corresponde a la Iglesia primitiva, aquella donde la figura física y la presencia real y sensible del Jesús histórico jugaba el papel primordial. Corresponde, dentro de la lectura conceptual que Hegel lleva a cabo, a la primera forma de conciencia inmediata, de conciencia sensible, en la que el objeto se hace presente de manera inmediata y singular al sujeto. A esta primera etapa sucede necesariamente una segunda, que implica una ruptura entre el objeto y el sujeto, un alejamiento, y que Hegel ve realizada en el catolicismo medieval. En esa etapa, la verdad ya no es algo inmediato, sino una realidad lejana situada en un objeto ajeno por completo a la conciencia, y esto en un doble sentido: primero, porque la verdad se presenta como una doctrina que debe aceptarse por una autoridad exterior, la Iglesia, que asume la administración de esa doctrina y de las costumbres que de ella se derivan; segundo, porque la figura de Cristo se pierde en el pasado y se anuncia para un futuro escatológico, y por ello se la percibe como ausente.

Este desgarramiento, que caracteriza al catolicismo, viene a ser un momento necesario, ya que solo a través de él aprende la conciencia del creyente y de la comunidad a relacionarse con la verdad ya no de manera sensible, sino conceptual, es decir, mediada por una verdadera reflexión. Entre el creyente y la palabra de Dios se introduce la Iglesia como único intérprete de esta, y la conciencia del católico pierde así toda autonomía tanto en lo doctrinal como en lo moral.

Este periodo de desgarramiento y formación debe dar paso a una nueva reconciliación, a una nueva identidad entre verdad y certeza, y esta reconciliación es obra de la Reforma protestante. Al quitar de en medio a la jerarquía eclesiástica y colocar al creyente de manera directa frente a la palabra de Dios, se recupera la absoluta libertad y se instaura un nuevo contexto o un nuevo paradigma para comprender las relaciones del ser humano consigo mismo, con la naturaleza, con sus semejantes y con Dios.

No es este el lugar para examinar en detalle la lectura que ofrece Hegel del fenómeno cristiano, y del catolicismo en particular, fenómeno que considera 
crucial para comprender no solo el mundo y la cultura moderna, sino también la filosofía que se ha venido desarrollando, así como su propia filosofía. En sus Lecciones sobre la filosofia de la historia universal, al analizar el llamado "tercer periodo" que corresponde a la modernidad (die neue Zeit), inicia con una exposición sobre la Reforma, a la que caracteriza como "el principio de la modernidad".

Sus primeras palabras señalan muy bien lo que habrá de decir a continuación:

Hemos llegado ahora al tercer periodo, el del reino germánico, e ingresamos con ello en el periodo del espíritu que se sabe libre en cuanto que quiere lo verdadero, lo eterno y lo universal en y para sí. En este periodo hay que hacer de nuevo tres divisiones. Primero tenemos que considerar la Reforma como tal, el sol que todo lo ilumina, y que sigue a aquel amanecer al final de la Edad Media; luego, el desarrollo de la situación después de la Reforma; y, finalmente, los tiempos recientes desde finales del siglo anterior. La Reforma surgió de la corrupción de la Iglesia. Para comprender esa lucha contra la Iglesia hay que leer algunos de los escritos de Lutero, ya que la Iglesia hoy en día ya no se halla en el estado contra el cual él luchó; también la Iglesia católica se ha purificado mediante la Reforma (Vorlesungen, 877).

En todo caso, lo que importa es recalcar el papel que el mensaje cristiano desempeña en la configuración de la libertad y de su manifestación en la organización de un Estado de derecho. Hegel no solo considera que una adecuada comprensión del cristianismo es clave para interpretar nuestro mundo actual, sino que ese fenómeno histórico juega un papel crucial cuando se trata de examinar las diversas posturas políticas que marcan la nueva situación de Europa.

Ahora bien, si nos mantenemos en ese esquema muy general de la historia del cristianismo que hemos esbozado antes, tenemos un primer periodo de identidad inmediata entre el creyente y su objeto de fe, un segundo periodo 
de desgarramiento, de escisión, de separación, y un tercer momento de reconciliación. La interpretación concreta de este tema, para lo que nos atañe, es decir, para la comprensión de los derechos humanos a la luz de la doctrina hegeliana, es que la experiencia dolorosa del catolicismo, que implicó una pérdida profunda de la libertad interior, ha sido un periodo de formación o de culturización. Bildung es el término alemán que Hegel emplea para expresarlo; término que podemos traducir también como "configuración". Esa formación consiste en configurar de tal manera el espíritu del creyente, que logre apartarse de sus sentidos y pueda así comprender el verdadero sentido de la doctrina.

Una religión que se haya elevado al nivel conceptual constituye, para Hegel, la condición indispensable para configurar una sociedad asentada sobre términos racionales. Y eso fue lo que no logró la Revolución francesa, por haberse instaurado en un contexto católico; y eso mismo es precisamente lo que Hegel espera que se configure en el terreno protestante de Alemania. En cierta forma, podemos decir que el catolicismo es para él el revulsivo que suscita en el espíritu humano su profunda convicción de ser libre. Libertad que debe ser conquistada, ya que no es simplemente un don de la naturaleza.

No se puede negar que Hegel dio muestras de una ingenua miopía histórica, cuando consideró que el luteranismo alemán era precisamente aquella forma de comprender y practicar el cristianismo que conduciría a los seres humanos hacia la configuración de un Estado regido por los criterios de la razón. Sin embargo, tal vez no haya sido tan ingenuo cuando señaló el papel esencial que habría de jugar la Reforma protestante en el nacimiento y la consolidación de las ideas liberales. Autores como Max Weber y Ernst Troeltsch han mostrado con buenas razones la influencia del protestantismo, sobre todo en sus versiones calvinista y anabaptista, en la configuración del capitalismo y de la democracia respectivamente.

Con lo dicho hasta aquí he tratado de exponer el esfuerzo de los idealistas alemanes, comenzando con Kant y siguiendo con Fichte y Hegel, para comprender ese fenómeno crucial de su época que fue la Revolución francesa y la 
instauración de regímenes democráticos en Europa. Hegel, sobre todo, buscó comprenderlo como el proceso mediante el cual la libertad era situada en la raíz misma de la organización social, y trató de comprender las conexiones nada sencillas que existen entre el carácter moral del fenómeno y su realización política. Como tuvimos ocasión de verlo, si bien los derechos humanos existen como tales con la sola existencia del ser humano, ellos no pueden llegar a ser efectivos sino gracias a su configuración en un Estado de derecho. Moral y política, a la vez que se diferencian, se hallan también firmemente conectadas, y no es posible lograr la primera sin instaurar la segunda.

Por otra parte, aunque el origen de esos fenómenos los halla Hegel en el mensaje de la revelación cristiana, considera que estos tienen un carácter universal precisamente en la medida en que se fundamentan en la razón, lo que explica su necesidad y su validez para todos. La razón, entonces, es el tribunal de última instancia ante el cual debe presentarse toda propuesta moral que tenga aspiraciones de tal, porque la razón no solo se conoce a sí misma y a todo lo que no es ella, sino que está en condiciones de fijar y determinar sus propios límites. Porque la razón tiene límites que si bien no son, para Hegel, los muy estrechos que le señaló Kant, apuntan, sin embargo, al carácter trascendente que tiene el sentido último de la existencia humana. Es cierto que el hombre posee su razón como único faro de orientación para su comportamiento tanto personal como social, pero esta misma razón le señala que si bien la realidad no tiene sentido sino por el ser humano y para el ser humano, ese sentido no lo da el ser humano, sino que lo descubre y se le hace presente, de modo que él puede actuar en beneficio del mismo. En esto consiste para Hegel la verdadera dignidad de los seres humanos. 